\title{
Understanding chronic stress: a narrative review of literature
}

\author{
Gayan Buddhika Senanayake $^{1^{*}}$ \& Carukshi Arambepola ${ }^{2}$ \\ ${ }^{1}$ Centre for Online Health, University of Queensland, Australia; ${ }^{2}$ Department of Community Medicine, Faculty of \\ Medicine, University of Colombo, Sri Lanka \\ *Correspondence: m.senanayake@uq.edu.au
}

DOI: https://doi.org/10.4038/jccpsl.v25i1.8196

Received on: 04 January 2019

Accepted on: 26 February 2019

\begin{abstract}
Though commonly used to describe the 'emotions' in daily life, stress is a scientifically valued concept which has slowly evolved since the late 1970s to become a vital area of research interest, expanding its scope in relation to humans, animals, and plants. In today's context, chronic stress is identified as one of the main determinants of physical and mental disorders.

The concept of chronic stress has a complex diversity and several multi-dimensional constructs. There had always been controversies on the mechanism and measurement of chronic stress, leading to confusion and disagreement among the scientific community, hence the slow progression in research related to chronic stress. These fundamental deficiencies and the emerging public interest were recognised in the early 1980s by the United States National Research Council and as a result, several organizations such as the International Institute of Stress in Montreal and Hans Selye Foundation were established to uplift the research work on stress. The first World Congress on stress was held in 1994 during which the current issues pertaining to stress were discussed extensively. A narrative review of the literature was conducted to explore the concept of chronic stress using several electronic databases.
\end{abstract}

Keywords: stress, chronic stress, narrative review

\section{Background}

'Stress' is one of the 'buzz words' of the modern world and has been labelled by the World Health Organization as the 'health epidemic of the $21^{\text {st }}$ century'. It is commonly overused or misused in everyday life as well as in biological science and clinical practice (1). 'Stress', derived from the English word 'distress' (2) denotes the interaction between humans and their environment at individual and community levels (3). It expresses not only the psychological, occupational and social pressures in life but also the lifestyles and health consequences (4). It is, therefore, identified as a modern metaphor of the darker side of human behaviour (3).

Acute stress is commonly used by the general public to describe 'time pressure' in day to day life. When a time-bound task cannot be accomplished, it 
could initiate a series of physiological reactions - a 'stress response'. However, only some would get 'stressed' by such time pressures, implying that stress has many other determinants specific to individuals for initiating a stress response (5). These determinants are external influences experienced by an individual or reactions of an individual with these forces, such as direct physical or chemical substance or indirect symbolic meanings that could interfere with the biological integrity of an individual to produce stress (6).

While acute stress is increasingly becoming part of life, 'chronic stress' has remained a relatively unfamiliar entity. Chronic stress is known to result in a variety of serious health related physical, financial and emotional consequences at both individual and societal levels (7-8). It is the responsibility of the public health researchers to unwind this complex scientific phenomenon into an applicable concept to improve disease prevention. Therefore, we aimed to conduct a narrative review on the concept of chronic stress based on literature.

\section{Methods}

A thorough literature review was performed to identify the facts related to chronic stress under the following themes: Evolution of stress, the concept of chronic stress, its patho-physiological basis, and health consequences. The electronic databases such as PubMed, Google Scholar, Medline, and HINARI were used to gather data. The search was limited to local and international literature published in English. The retrieved literature was checked for secondary references in order to ensure a comprehensive search. The search strategy included all aspects of chronic stress: evolution, concept, patho-physiology and health implications of chronic stress. Medical subject headings (MeSH) terms were used when searching Medline. For the other databases, the Boolean operators 'and' and 'or' were utilized. Manual search for relevant literature was also done in hard copies of medical journals in libraries, theses, and dissertations. Citation information along with notes and abstracts were downloaded to Endnote X6 software.

\section{Evolution of stress}

Stress as a concept came to the fore in the late 17 th century and started evolving based on science only in the early $19^{\text {th }}$ century (9). In the last 25 years, it has become a vital area of research gaining due recognition in the world.

The word "stress" has been used initially by engineers to describe the force that can put a strain on a particular structure. The maximum force that can be applied to a given structure was known as its 'stress threshold' (5). In the early $20^{\text {th }}$ century, Walter Cannon who did research on adrenal medulla formulated a hypothesis on stress related to humans. He hypothesized that activation of the autonomic nervous system (mainly the sympathetic system) prepares an individual to produce a 'fight or flight' response to danger (10). This hypothesis was challenged by Hans Selye in 1935, based on his experiments conducted on laboratory rats. In his experiments, he administered a variety of ovarian and placental extracts that were considered as stressful agents' into rats and observed their organ-specific changes. He observed the following pathological changes in all the animals: ulceration of intestines, atrophy of the thymus and lymphatic system and ulceration of the adrenal cortex. Based on this, Selye proposed that this triad was a consistent reaction to any demand and not only to danger (3). This led to re-defining of stress as 'a nonspecific (stereotypical) response of the body to any demand' (11). To express 'demands', he coined a new term called 'stressors', which has the potential to act as internal or external agents to produce a stress response. Along with it, Selye constructed the 'General Adaptation Syndrome' (GAS) related to homeostasis. Homeostasis is a 'steady state' where all living organisms maintain their equilibrium that is essential for survival (12). This equilibrium is shown to be affected by stressors (13). GAS leads to adaptation, which is the physical or mental response that is needed to regain homeostasis (14). If no such adaptation takes place, it leads to a 'stress response' in an individual (15).

The GAS consists of three stages: alarm reaction, resistance, and exhaustion. Alarm reaction consists of two sub-components: shock and counter-shock. The shock phase is controlled using the neural mechanisms that stimulate the adrenal medulla. This stimulation also releases several hormones during this phase. The counter-shock phase reverses the changes occurring during the shock phase, while the stage of resistance is consistent with increased parasympathetic activity. 
High secretion of glucocorticoids and increased anabolism of proteins are the main features during this stage. If a person enters the stage of exhaustion, the exposure to stressors may damage the adaptation process leading to various other changes (15).

In the early 1960s, John Mason - a psychologist measured the level of stress hormone among subjects who were exposed to different stressors. He proposed that stress experienced is almost always the result of a specific cognitive mediation, in which the stimulus must be appraised as a threat for it to produce a stress response. Mason and his research team had enough evidence to challenge Selye and to conclude that the response to stress is specific, unlike what Selye described as stereotypical (16).

The concept of stress has been further refined by understanding the pathophysiology of the stress response. Everly and Sobelman (17) identified three neuro-biological pathways of the stress response, which are neurologic, neuroendocrine and endocrine trails. Several other conceptual frameworks have been proposed and as a result, several methodological issues have arisen in relation to the study of stress. This has made the subject of stress more complex and challenging (11).

\section{The concept of chronic stress}

The concept of chronic stress was initially evaluated using animals as experimental models. These animals were exposed to external stressors for a long period of time, thus the chronicity of stress was mainly determined by the duration of stressors $(11,18)$. This has been challenged in subsequent research.

The mechanism of acute and chronic stress is not the same. This difference has been explained by the different stages of GAS. Acute stress mainly corresponds to the alarm and resistant phases of reaction, whereas exhaustion directly deals with the concept of chronic stress. To further elaborate, during an acute traumatic event, the individual will enter into the alarm phase with warning signs. To face this event, the body will take safety measures during the resistance phase. Some may have few or no damages related to this incident, which results in a successful adaptation. On the other hand, some stressors, for example, severe injury or death of a loved one would continue to discharge a stress response, even after the termination of the physical event. After a considerable time, they would go into the exhaustion phase (11). This persisting response is identified as 'chronic stress'.

Not only the duration of resistance stages but also the frequency of the alarm or resistance is identified as an indicator to differentiate acute stress from chronic stress. Individuals exposed to repeated stressors may circulate back and forth between alarm and resistance stages. This cycling effect is facilitated by the replacement of corticosteroids with continuous discharge of adrenal hormone. When the chronic stressor lasts long enough, the hormone discharge may tail-off and the exhaustion begins, leading to chronic stress. It should be noted that a stressful event or stimulus needs to fulfil several other factors to produce chronic stress. Duration of the stressful event, the uncertainty of stress, loss of adaptation to demands or threat are some of them (11).

A conceptual confusion has been noted in differentiating chronic stress from daily hassles (19). Daily hassles are "irritating, frustrating, distressing demands that characterize everyday transactions with the environment" (19), whereas chronic stress is also due to high- or low-intensity stressors with a high frequency of occurrence (19). This confusion has been resolved to some extent by Pratt and Barling (20), in which they identify daily hassles as annoying clusters of events that are of low intensity as well as occurring infrequently, compared to chronic stressors occurring frequently in high- or low-intensity.

In further support of the theory of chronic stress, McEwen introduced the concept of 'allostatic load' in 1993, which is the long-term effect of the physiological response to stress. In simple terms, it is the number of stress response cycles that a person would undergo. Large allostatic loads are detected due to several reasons: an environment that causes repeated activations, lack of adaptation to environmental stressors or inability to decline towards the baseline (21). Allostasis is the adaptive mechanism used to achieve homeostasis by producing mediators such as adrenaline, cortisol and other chemical messengers (22).

In 2013, Smyth \& colleagues (23) have demonstrated a conceptual model for chronic stress. According to this model, three contributing factors are identified as giving rise to chronic stress namely, repeated activation, low or slow adaptation, and delay 
or failure to return to homeostasis. He proposes that chronic stress and associated health risk occur when the stress response is "too often" or "too long", which in turn produces a sustained effect on biological systems.

Repeated activation or higher frequency of acute stressors: This may be the same stressor or another stressor arising from the environment that is related or unrelated to the previous one. It is not necessary to have the external stressor all the time. An individual can perceive the challenging/threatening event repeatedly so that it generates a chronic stress response (23). The schematic illustration given in Figure 1 differentiates the stress response of a person with chronic stress and of a normal person. According to Figure 1, line ' $A$ ' shows how the body should ideally respond to repeated stressors. With time, the stress response to repeated stressors will be of less intensity and will reach homeostasis, although it may not reach a zero response. This is an acceptable response of a normal person. Line ' $\mathrm{B}$ ' explains the stress response of a person with chronic stress, in which every time the stressor comes, he is reacting to it as if for the first time in full force without any habituation with time. Importantly, if that person is removed from this environment, he/she can function normally again.

Low or slow adaptation: This contributes to the lack of habituation with persistent physiological responses even for non-threatening stressors. Continuous appraisal of stressors as threatening is the main mechanism of action. Duration of both the stressor and its response contribute to this low/ slow adaptation (23).

Delay or failure to return to homeostasis: This is extra time that needs an individual to return to homeostasis or may not come to the usual homeostasis status and settles at a much higher resting state. Persistent stress responses occur due to complex environmental challenges which need plenty of biological resources, thus leading to slower recovery (24). The continuation of the stress state for a long period of time contributes to chronic stress (23).

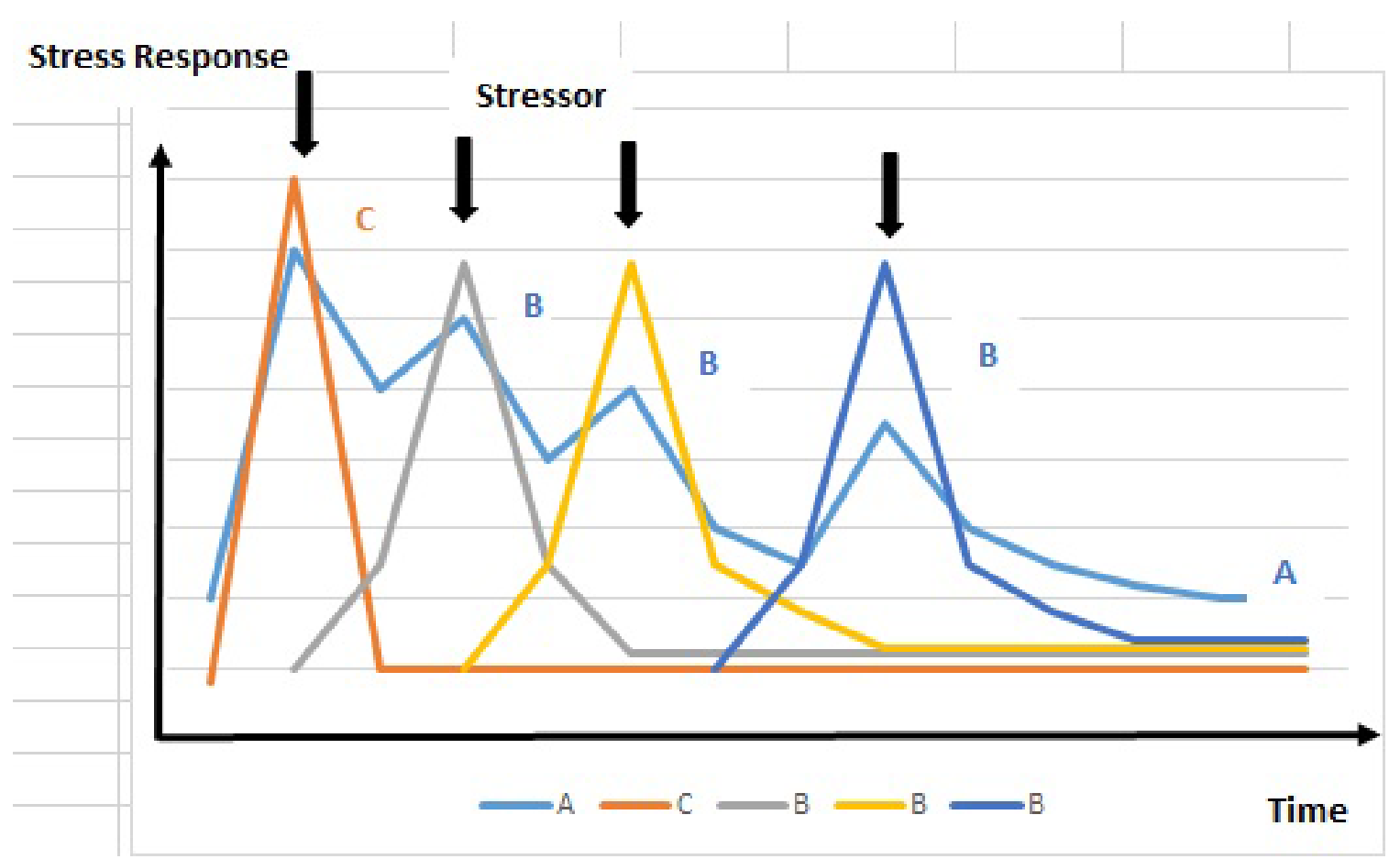

Figure 1. Schematic illustration of the stress response of a person with chronic stress and of a normal person. 


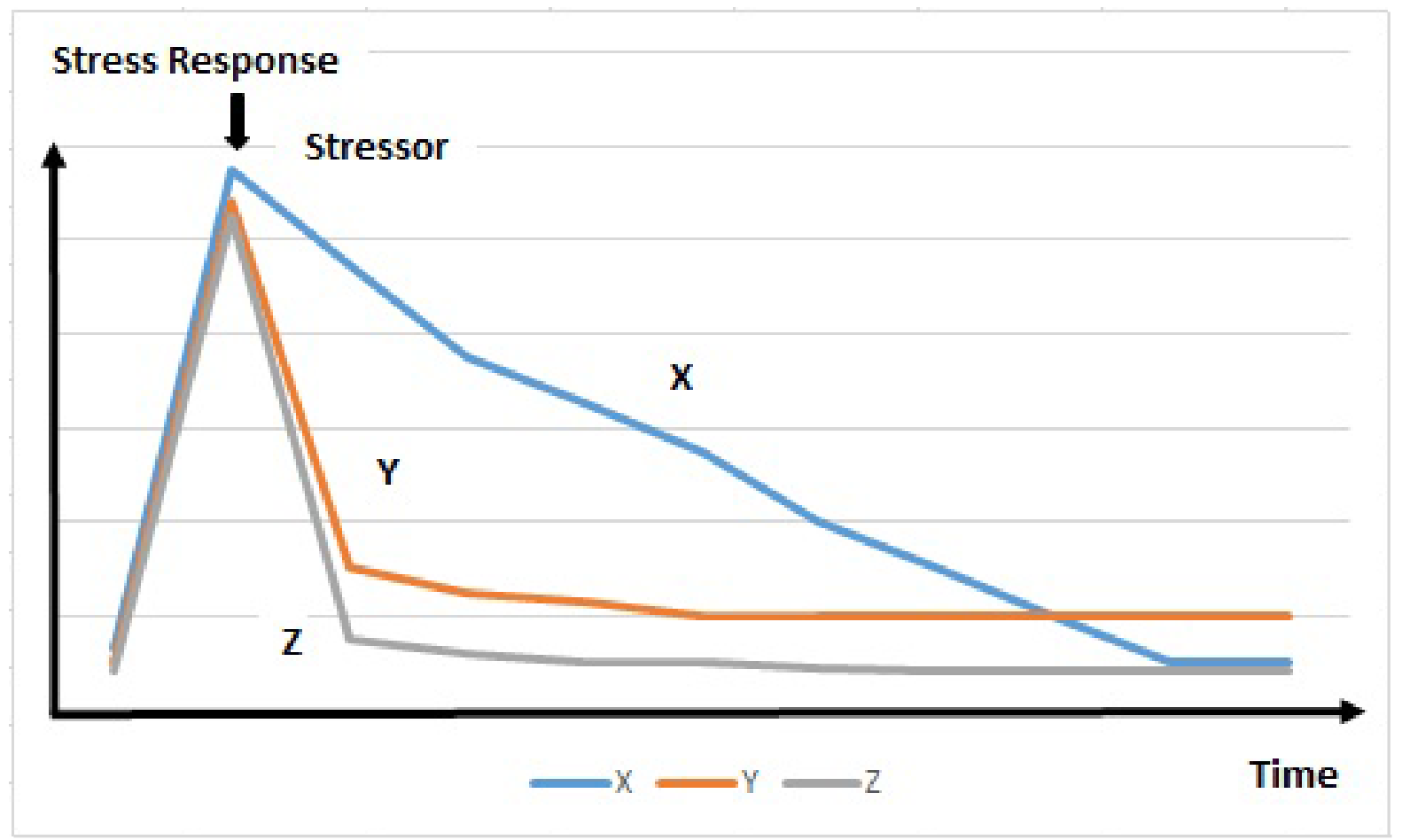

Figure 2. Schematic illustration of abnormal stress response and normal stress response.

The schematic illustration given in Figure 2 differentiates the abnormal stress response (inline ' $\mathrm{X}$ ' and ' $\mathrm{Y}$ ') from the normal stress response (inline ' $\mathrm{Z}$ '). The abnormal responses may be due to inherent properties of the person (e.g. personality) and may not have any relationship with the external environment, so that even if their environment is changed, the way they respond to stress may not differ.

Based on these concepts, operational definitions for chronic stress have been derived. Eckenrode defines chronic stress as "aspects of the environment that are demanding an ongoing and relatively unchanging basis" (25). Chronic stress is also defined as "repeated occurrence of different stressors with uncontrollable consequences or absence of adaptive coping mechanisms" (20, 24).

According to Baum (18), chronic stress is "demands, threats, perceived harm or loss, or responses that persist for a long period of time". Tate and others (26) have defined chronic stress as "lasting for more than two weeks without specific dates that stand out as particularly significant" such as the presence of financial difficulties after losing the job. Wheatley (27) has operationally defined chronic stress as "an event/ stressor of high intensity that occurred more than once over a period of time" in her study on personal features, chronic stress and depressive symptoms. Hamilton and Meston (28) defined chronic stress in two components. The first component includes major life events with high intensity that lasts for a long period of time, such as the death of a loved one. The second component consists of a collection of small frequent stressors such as financial issues.

According to the literature, the definitions on chronic stress are more focused on 'stressors' rather than the 'stress response'. This highlights the need for a comprehensive definition, which should capture the stress response and the interaction between a person and his/her environment. The importance of stress response in developing chronic stress is emphasized in the pathogenesis of chronic stress.

\section{Pathophysiology and health consequences of chronic stress}

The acute stress response increases the activity of the hypothalamo-pituitary adrenal (HPA) axis, resulting in high levels of cortisol. It has been noted that chronically stressful events can increase or 
decrease the body cortisol output (29-31). This chronic stress-induced hyper- and hypo-cortisolism has created confusion regarding the exact mechanism of HPA activity. To clarify this, Miller and others (32) conducted a meta-analysis using available research data over the past fifty years. Results of this meta-analysis highlighted that chronic stress has the ability to increase or decrease the HPA activity, while it depends on the characteristics of stress and the individual who faces stress. The review identified five key characteristics that shape the HPA activity following an individual's exposure to chronic stress. These include the time since the onset of chronic stress, the nature of the threat, emotions elicited by stress, controllability of stress and the individual psychiatric sequelae.

An acute stress response consists of an emotional response, followed by coping strategy and defence mechanism. All three components prevent a person moving towards a disease status (33). In contrast, chronic stress causes permanent and long-term damage to physical as well as mental health through alterations in physiological, behavioural and psychological responses of the systems and organs of the body (24).

Physical and mental disorders are closely related to stressors in life (34). A stressor can disturb or threaten the normal homeostasis mechanism of the two main stress response pathways: HPA stress axis and sympathetic-adrenal-medullary (SAM) system (34). Continued and prolonged chronic stressors disturb the HPA axis and its negative feedback mechanism that leads to maladaptation of this axis. This is also known as "adrenal fatigue" (35). The final result is the high glucocorticoid hormone levels, altered release of hypothalamic hormones and reduction of negative feedback control of the two axes (36). Chronic stress activates the SAM system and releases catecholamines to initiate the response. This continuous and long-term activation of HPA and SAM systems increases the risk of physical and mental disorders (34).

Chronic stress is directly interrelated with stress endocrine system and affects individual brain structure, immune system and behaviour. This clearly shows that individuals who are exposed to chronic stress during their life spans are at higher risk of developing cardiovascular disease, obesity, cancer, immune disorders and mental disorders such as depression (37-38).

\section{Conclusions}

Repeated stressors can produce a stress response in full force, in which the system responding to stress (i.e. the pathway to come back to homeostasis) wears out. Ultimately, the stress response will remain high giving rise to chronic stress. When the body fails to reach homeostasis as a consequence of the chronic stress response, other organs and systems in the body would start changing, which would lead to physical and mental disease entities.

\section{References}

1 Charlton BG. Stress. Journal of Medical Ethics 1992; 18(3): 156-159.

2. National Research Council (US) Committee on Recognition and Alleviation of Distress in Laboratory Animals. Recognition and alleviation of distress in laboratory animals. Washington (DC): National Academies Press (US), 2008.

3. Viner R. Putting stress in life: Hans Selye and the making of stress theory. Social Studies of Science 1999; 29(3): 391-410.

4. Hogan C. Chronic stress. An approach to management in general practice. Australian Family Physician 2013; 42:542-545.

5. Centre for Studies on Human Stress. How to measure stress in humans? Quebec: Fernand-Seguin Research Centre of Louis-H, Lafontaine hospital, 2007.

6. Stults-Kolehmainen MA \& Sinha R. The effects of stress on physical activity and exercise. Sports Medicine 2014; 44(1): 81-121.

7. Schmidt MV, Sterlemann V, Muller MB. Chronic stress and individual vulnerability. Annals of the New York Academy of Sciences 2008; 1148: 174-183.

8. Davis MT, Holmes SE, Pietrzak RH, Esterlis I. Neurobiology of chronic stress-related psychiatric disorders: evidence from molecular imaging studies. Chronic Stress (Thousand Oaks) 2017; 1. DOI: 10.1177/2470547017710916.

9. Robinson AM. Let's talk about stress: history of stress research. Review of General Psychology. 2018. DOI: 10.1037/gpr0000137.

10. Stefan Bracha H, Ralston TC, Matsukawa JM, Williams AE, Bracha AS. Does "fight or flight" need updating? Psychosomatics 2004; 45(5): 448-449.

11. O'keeffe MK \& Baum A. Conceptual and methodological issues in the study of chronic stress. Stress Medicine 1990; 6: 105-115.

12. Chrousos GP. Stress and disorders of the stress system. Nature Reviews Endocrinology 2009; 5(7): 374-381. 
13. Susman EJ, Nottelmann ED, Dorn LD, Inoff-Germain G, Chrousos GP. Physiological and behavioral aspects of stress in adolescence. In: Chrousos GP, Loriaux DL, Gold PW (Eds.) Mechanisms of physical and emotional stress. Advances in experimental medicine and biology, vol 245. Springer: Boston, MA, 1988.

14. Chrousos GP \& Gold PW. The concepts of stress and stress system disorders: overview of physical and behavioural homeostasis. Journal of the American Medical Association 1992; 267(9): 1244-1252.

15. Larzelere MM \& Jones GN. Stress and health. Primary Care 2008; 35(4): 839-856.

16. Trotter RJ. Stress: confusion \& controversy. Science News 1975; 107(22): 356-359.

17. Everly G \& Sobelman S. Assessment of the human stress response. New York: AMS Press, 1987.

18. Baum A. Stress, Intrusive Imagery and chronic distress. Health Psychology 1990; 9(6): 653-675.

19. Hahn SE \& Smith CS. Daily hassles and chronic stressors: conceptual and measurement issues. Stress Medicine 1999; 15: 89-101.

20. Pratt LI \& Barling J. Differentiating between daily events, acute and chronic stressors: a framework and its implications. In: Hurrell JJ, Murphy LR, Sauter SL, Cooper CL (Eds.), Occupational Stress: Issues and Development in Research. New York, Philadelphia, London: Taylor \& Francis, 1988.

21. McEwen BS. Neurobiological and systemic effects of chronic stress. Chronic stress (Thousand Oaks) 2017; 1. DOI: $10.1177 / 2470547017692328$.

22. McEwen BS. Stressed or stressed out: What is the difference? Journal of Psychiatry and Neuroscience 2005; 30(5): 315-318.

23. Smyth J, Zawadzki M, Gerin W. Stress and disease: a structural and functional analysis. Social and Personality Psychology Compass 2013; 7(4): 217-227.

24. McEwen BS. Protective and damaging effects of stress mediators. New England Journal of Medicine 1998; 338(3): 171-179.

25. Eckenrode J. Impact of chronic and acute stressors on daily reports of mood. Journal of Personality and Social Psychology 1984; 46: 907-918.

26. Tate SR, Brown SA, Glasner SV, Unrod M, Mcqaid JR. Chronic life stress, acute stress events, and substance availability in relapse. Addiction Research \& Theory 2006; 14(3): 303-322.
27. Wheatley MA. Personal characteristics, chronic stress and depressive symptoms in the life of AfricanAmerican women. PhD Thesis. Case Western Reserve University, School of Graduate Studies, 2009.

28. Hamilton LD \& Meston CM. Chronic stress and sexual function in women. The Journal of Sexual Medicine 2013; 10(10): 2443-2454.

29. Bowirrat A, Chen TJH, Blum K, Madigan M, Bailey JA, Chuan Chen AL, et al. Neuro-psycho-pharmacogenetics and neurological antecedents of posttraumatic stress disorder: unlocking the mysteries of resilience and vulnerability. Current Neuropharmacology 2010; 8(4): 335-358.

30. Seedat S, Stein MB, Kennedy CM, Hauger RL. Plasma cortisol and neuropeptide $\mathrm{Y}$ in female victims of intimate partner violence. Psychoneuroendocrinology 2003; 28(6): 796-808.

31. Vedhara K, McDermott MP, Evans TG, Treanor J, Plummer S, Tallon D, et al. Chronic stress in nonelderly caregivers. Psychological, endocrine and immune implications. Journal of Psychosomatic Research 2002; 53: 1153-1161.

32. Miller GE, Chen E, Zhou ES. If it goes up, must it come down? Chronic stress and the hypothalamic-pituitaryadrenocortical axis in humans. Psychological Bulletin 2007; 133(1): 25-45.

33. Stephens MAC, Wand G. Stress and the HPA axis: role of glucocorticoids in alcohol dependence. Alcohol Research 2012; 34(4): 468-483.

34. Cohen S, Janicki-Deverts D, Miller GE. Psychological stress and disease. Journal of American Medical Association 2007; 298(14): 1685-1688.

35. Wilson JL. Adrenal fatigue: the $21^{\text {st }}$ century stress syndrome. Petaluma: Smart Publication, 2001.

36. Blackburn-Munro G, Blackburn-Munro RE. Chronic pain, chronic stress and depression: coincidence or consequence? Journal of Neuroendocrinology 2001; 13(12): 1009-1023.

37. Cohen S, Janicki-Deverts D, Miller GE. Psychological stress and disease. Journal of the American Medical Association 2007; 298(14): 1685-1687.

38. Matosin N, Cruceanu C, Binder EB. Preclinical and clinical evidence of DNA methylation changes in response to trauma and chronic stress. Chronic stress (Thousand Oaks) 2017; 1: 10. DOI: 1177/ 2470547017710764. 